\title{
Bottleneck-induced transitions in a minimal model for intracellular transport
}

\author{
Paolo Pierobon, ${ }^{1}$ Mauro Mobilia, ${ }^{1}$ Roger Kouyos, ${ }^{2}$ and Erwin Frey ${ }^{1}$ \\ ${ }^{1}$ Arnold Sommerfeld Center for Theoretical Physics and Center for NanoScience, Department of Physics, \\ Ludwig-Maximilians-Universität München, Theresienstrasse 37, D-80333 München, Germany \\ ${ }^{2}$ Theoretische Biologie, Eidegenössische Technische Hochschule Zürich, Universitätstrasse 16, CH-8092 Zürich, Switzerland
}

(Received 13 April 2006; published 13 September 2006)

\begin{abstract}
We consider the influence of disorder on the nonequilibrium steady state of a minimal model for intracellular transport. In this model particles move unidirectionally according to the totally asymmetric exclusion process (TASEP) and are coupled to a bulk reservoir by Langmuir kinetics. Our discussion focuses on localized point defects acting as a bottleneck for the particle transport. Combining analytic methods and numerical simulations, we identify a rich phase behavior as a function of the defect strength. Our analytical approach relies on an effective mean-field theory obtained by splitting the lattice into two subsystems, which are effectively connected exploiting the local current conservation. Introducing the key concept of a carrying capacity, the maximal current that can flow through the bulk of the system (including the defect), we discriminate between the cases where the defect is irrelevant and those where it acts as a bottleneck and induces various novel phases (called bottleneck phases). Contrary to the simple TASEP in the presence of inhomogeneities, many scenarios emerge and translate into rich underlying phase diagrams, the topological properties of which are discussed.
\end{abstract}

DOI: 10.1103/PhysRevE.74.031906

PACS number(s): 87.10.+e, 02.50.Ey, 05.60.-k, 64.60.-i

\section{INTRODUCTION}

The effects of disorder on the phase behavior have been investigated in a multitude of statistical mechanics models and there is by now a good understanding of the ensuing equilibrium phenomena $[1,2]$. In contrast, in nonequilibrium statistical mechanics, the effect of disorder on dynamics and nonequilibrium steady state is far from being well understood [3].

One of the best studied cases is the totally asymmetric simple exclusion process (TASEP) [4-20]. It is defined as a one-dimensional lattice gas where particles are hopping stochastically in one direction subject to hard-core repulsion (for reviews see Refs. [21,22]). Two types of disorder have been studied: hopping rates may either depend on the particle attempting to jump (particle-wise disorder [4-6] or each lattice site may be associated with a random quenched hopping rate (site-wise disorder) [7-20]. As intuitively expected, such defects generally induce phase separations if the particle traffic exceeds a certain threshold [6].

Our studies are motivated by an important class of intracellular transport processes mediated by molecular motors like kinesin, dyneins, or myosins moving along cytoskeletal filaments like microtubules or F-actin [23]. The dynamics of each of these molecular engines is a complicated stochastic process, which we idealize by a Poisson process with a single rate limiting step only. There is now convincing evidence that transport along the cytoskeletal filaments is onedimensional and binding sites are periodically spaced [24]. Since each of the binding sites can be occupied by at most one of the molecular motors, particle exclusion can play a crucial role. Though there is no clear evidence in vivo, at the moment, that motor densities are large enough for particle exclusion to dominate the transport properties, there are in vitro investigations underway studying the transport of kinesin along microtubules at high-volume concentrations [25]. Each molecular track has a finite length, and in general one would like to allow for the enzyme reservoirs at both ends to have different densities and/or attachment rates at the left and right end to be different from each other and different from the hopping rate in the bulk. All this taken together defines the TASEP, introduced originally as a model for the kinetics of biopolymerization on a nucleic acid template $[26,27]$. For it to be a proper minimal model for molecular intracellular transport it has also to account for the fact that microtubules are embedded in the cytosol with a reservoir of motors in solution. This allows for motors to attach from the solution to the molecular track or detach from it and become part of the reservoir again [28]. Then one arrives at the TASEP with Langmuir kinetics (TASEP/LK) introduced in Ref. [29], which exhibits phase separation even in the absence of any defects.

In this paper we would like to study the effect of an isolated defect on the nonequilibrium steady state of this minimal model for intracellular transport. We focus on a site-wise disorder that may be mediated by structural imperfections of the microtubular structure or proteins associated with the microtubules that change the affinity of the motors with the track. There has been evidence that these microtubule associated proteins might even be responsible for some diseases connected to motor proteins [30,31].

Let us now introduce the model under investigation. We consider a simple exclusion process on a finite lattice with $N$ sites (labeled $i=1, \ldots, N$ ), where the occupation number $n_{i}$ of each site can be either 0 or 1 . The dynamics of the system is described by a fully unidirectional continuum stochastic process in which each particle jumps randomly with rate $r_{i}$ to its right-neighboring vacant site. At the left, boundary particles are introduced in the lattice with rate $\alpha$, while at the right boundary they are extracted with rate $\beta$. This defines what is known as totally asymmetric simple exclusion process (TASEP) for $r_{i}=r$ (we define the time scale by setting $r=1$ ). We supplement this process in two ways, as shown in Fig. 1: (a) the system is coupled to a bulk reservoir via Langmuir kinetics; namely, particles can attach to a site in the bulk with 


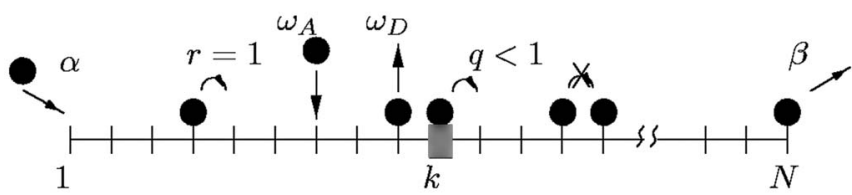

FIG. 1. Schematic representation of the TASEP with on-off kinetics in the presence of a bottleneck at the site $i=k$ (thick tick). The allowed moves are: forward jump (with rate $q \neq 1$ in $i=k$ and $r=1$ elsewhere), entrance at the left boundary (with rate $\alpha$ ), exit at the right boundary (with rate $\beta$ ), attachment (with rate $\omega_{A}$ ), and detachment (with rate $\omega_{D}$ ) in the bulk.

rate $\omega_{A}$ and can detach with rate $\omega_{D}[32] ;$ (b) a special site $k$ of the system represents a bottleneck and has a slower hopping rate $r_{k}=q<1$ [33]. Both cases have been separately studied previously and show nontrivial features. In this work we investigate the combined effect of these two perturbations to the usual TASEP: our study concerns a detailed analysis of the nonequilibrium steady-state properties, with emphasis on the resulting phase diagrams. Two essential ingredients to carry on such an analysis are the local density of particles $\rho_{i} \equiv\left\langle n_{i}\right\rangle$ and current profile $j_{i} \equiv r_{i}\left\langle n_{i}\left(1-n_{i+1}\right)\right\rangle$; where the brackets stand for the average over the histories. Following the same steps as in Refs. [29,34] (see also Ref. [35] for a recent review), the stationary density is shown to obey a hierarchy of equations involving nearest-neighbor correlation functions

$$
\begin{gathered}
0=r_{i-1}\left\langle n_{i-1}\left(1-n_{i}\right)\right\rangle-r_{i}\left\langle n_{i}\left(1-n_{i+1}\right)\right\rangle \\
+\omega_{A}\left\langle 1-n_{i}\right\rangle-\omega_{D}\left\langle n_{i}\right\rangle, \quad i=2, \ldots, N, \\
0=\alpha\left\langle 1-n_{1}\right\rangle-\left\langle n_{1}\left(1-n_{2}\right)\right\rangle, \\
0=\left\langle n_{N-1}\left(1-n_{N}\right)\right\rangle-\beta\left\langle n_{N}\right\rangle,
\end{gathered}
$$

where, to account for the defect at site $i=k$, we have

$$
r_{i}= \begin{cases}1, & \text { if } i \neq k \\ 0<q<1, & \text { if } i=k .\end{cases}
$$

To be sure to capture an interesting interplay between boundary-induced phenomena and bulk dynamics (see Refs. $[29,34,36])$ we shall consider a mesoscopic limit where local adsorption-desorption rates are rescaled such that the gross rates $\Omega_{A, D}$ are comparable to the injection-extraction rates at the boundaries

$$
\Omega_{D}=N \omega_{D}, \quad \Omega_{A}=N \omega_{A} .
$$

Keeping the gross rates fixed in the limit $N \rightarrow \infty$ introduces interesting competition between boundaries and bulk.

This work is organized as follows: Sec. II summarizes the main properties of the TASEP, TASEP coupled to the Langmuir kinetics, and TASEP with a single inhomogeneity. In Sec. III, we outline the effective theory on which we build our analysis. Section IV is devoted to the discussion of our results (phase diagrams and density profiles). Finally, we present our conclusions in Sec. V.

\section{REVIEW OF PREVIOUS RESULTS: SEPARATE ROLE OF ON-OFF KINETICS AND DEFECT}

The effective theory presented hereafter is built on the properties of models akin to the one under consideration here, namely, the TASEP and TASEP coupled to Langmuir kinetics. It is thus appropriate to briefly review the main features of the latter and to summarize the results for the simple TASEP in the presence of a single inhomogeneity.

\section{A. The TASEP}

In the absence of attachment and detachment and with uniform hopping rate, the model defined above reduces to the TASEP. Much is known about the nonequilibrium steady state of this paradigmatic model. Both exact methods [37-41] and approximated mean-field solutions $[26,27,42,43]$ show that as a function of the entrance and exit rates there are three distinct nonequilibrium steady states. For $\beta<1 / 2$ and $\alpha>\beta$ there is a high-density phase (HD), where both the density and the current are determined by the exit rate $\beta$. Mean-field theory gives a spatially constant density $\rho_{i}=1-\beta$ larger than $1 / 2$ and a constant current $j_{i}=\beta(1-\beta)$. Thus, the current is dominated by the low exit rate, which acts as a bottleneck for the transport.

In contrast, for $\alpha<1 / 2$ and $\alpha<\beta$, the low entrance rate is the limiting factor for the particle current, which is now given by $j_{i}=\alpha(1-\alpha)$. Since $\rho_{i}=\alpha$ is always smaller than $1 / 2$ in this parameter range, the phase is also termed the lowdensity (LD) phase.

If both $\alpha$ and $\beta$ become larger than the critical value $1 / 2$ the density becomes constant $\rho^{*}=1 / 2$ independently of the parameters at the boundaries. The current is limited by the particle exclusion in the bulk and its maximal value is $j^{*}$ $=1 / 4$; therefore, this phase was termed the maximal-current (MC) phase.

\section{B. The TASEP with on-off kinetics}

Supplementing the TASEP, a genuine driven (nonequilibrium) process, with on-off (or Langmuir, equilibrium) kinetics results in a system termed TASEP/LK. If the rates of the Langmuir kinetics are faster than, or comparable to, the hopping rates, the on-off kinetics always dominates the driven process. To guarantee the particles to cover a relevant portion of the lattice before detaching, the mesoscopic limit mentioned in Eq. (5) has been introduced [29]. This imposes a time scale where competition between the boundary, driven processes, and the on-off kinetics, is effective and results in rich collective phenomena.

Considering Eqs. (1)-(3) with $r_{i}=1$, the mean-field analysis simply consists of neglecting any spatial correlations resulting in the following decoupling approximation:

$$
\begin{gathered}
\left\langle n_{i} n_{i+1}\right\rangle \approx\left\langle n_{i}\right\rangle\left\langle n_{i+1}\right\rangle=\rho_{i} \rho_{i+1}, \\
j_{i} \approx\left\langle n_{i}\right\rangle\left(1-\left\langle n_{i+1}\right\rangle\right)=\rho_{i}\left(1-\rho_{i+1}\right) .
\end{gathered}
$$

Taking the continuum limit with the new spatial variable 0 $\leqslant x \equiv i / N \leqslant 1$ and using the mesoscopic limit of Eq. (5) one obtains 

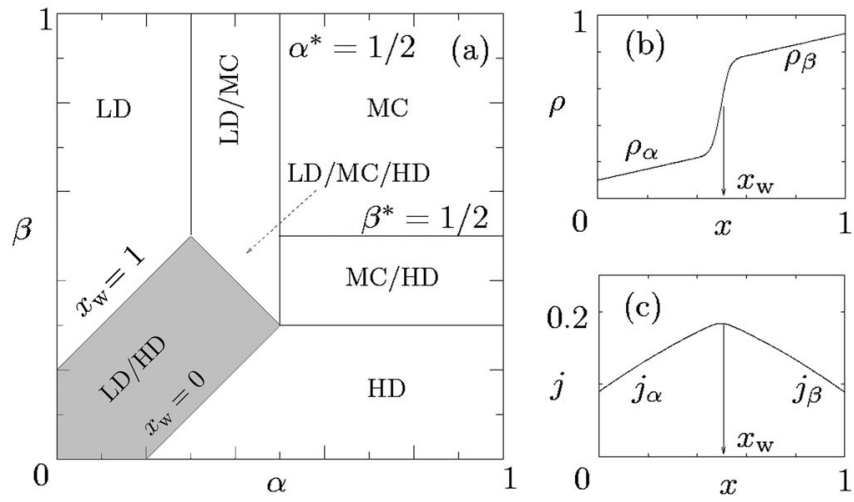

FIG. 2. (a) Phase diagram of TASEP/LK for $K=1$. One recognizes seven phases: in addition to the TASEP LD, HD, and MC phases, there are four more coexistence phases, namely, the LD/HD, $\mathrm{LD} / \mathrm{MC}, \mathrm{MC} / \mathrm{HD}$, and LD/MC/HD phases. The shaded region highlights the LD/HD coexistence where a localized domain wall appears. (b) Typical density profile in the LD/HD phase and (c) the corresponding current profile. At the matching point $x_{w}$ between the left $\left(j_{\alpha}\right)$ and right $\left(j_{\beta}\right)$ currents a domain wall develops and connects the left $\left(\rho_{\alpha}\right)$ and right $\left(\rho_{\beta}\right)$ density profile.

$$
(2 \rho-1) \partial_{x} \rho-\Omega_{D} \rho+\Omega_{A}(1-\rho)=0 .
$$

For the sake of simplicity, let us consider the case where the two rates are the same $\left(\Omega_{D}=\Omega_{A}=\Omega\right)$. In this case, Eq. (8) reads

$$
(2 \rho-1)\left[\partial_{x} \rho-\Omega\right]=0 .
$$

This equation obviously has two solutions: a linear one with slope $\Omega$ and a constant one coinciding with the critical density of the TASEP

$$
\rho(x)=\Omega x+C \quad \text { and } \quad \rho(x)=\rho^{*}=\frac{1}{2},
$$

where $C$ is a constant to be determined by the boundary conditions. The linear density profile results in a spacedependent current, which in mean-field reads

$$
j[\rho(x)]=\rho(x)[1-\rho(x)] .
$$

Equation (9) has to be supplemented by the boundary conditions

$$
\rho(0)=\alpha, \quad \rho(1)=1-\beta .
$$

When the solution of Eq. (10) cannot be matched continuously with the left and right boundaries (12), the density profile displays a localized discontinuity (or shock) in the bulk. This translates into the emergence of mixed phases. The latter are discussed in Refs. [29,34] and are summarized in the TASEP/LK phase diagram of Fig. 2(a). Let us illustrate this concept by considering the transition from the LD to the LD-HD phase. In the former, the density is determined by the left boundary and reads

$$
\rho(x)=\rho_{\alpha}(x) \equiv \Omega x+\alpha .
$$

Lowering the exit rate $\beta$ (with fixed $\alpha$ ), there is a site where both the currents imposed by the left and the right boundaries meet at $x_{w}$ [see Fig. 2(c)]. This is a mixed LD and HD
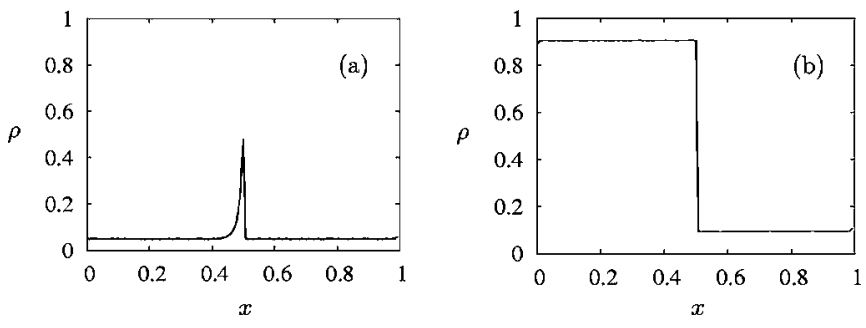

FIG. 3. Transition between a spike (a) and a step (b) in the density profile for the TASEP with defect. Stochastic simulations are performed on systems of size $N=128$ with parameters $q=0.1$, $x_{d}=0.5$, (a) $(\alpha, \beta)=(0.05,0.8)$, (b) $(\alpha, \beta)=(0.8,0.8)$.

phase. As shown in Fig. 2(b), the density has a sharp phase boundary (shock) at $x_{w}$,

$$
\rho(x)= \begin{cases}\rho_{\alpha}(x) \equiv \Omega x+\alpha, & \text { for } 0<x<x_{w} \\ \rho_{\beta}(x) \equiv 1-\beta+\Omega(x-1), & \text { for } x_{w}<x<1 .\end{cases}
$$

Another feature of the TASEP/LK is the particle-hole symmetry, i.e., the properties of the system are invariant under the exchanges $\alpha \leftrightarrow \beta, x \leftrightarrow 1-x$, and $\rho \leftrightarrow 1-\rho$. This translates in Fig. 2, which is symmetric with respect to the line $\alpha=\beta$ [29].

The case $\Omega_{A} \neq \Omega_{D}$ follows along the same lines but is mathematically more involved; for its treatment we refer to Ref. [34]. In the following sections we will consider $\Omega_{A}$ $=\Omega_{D}=\Omega$ and present the case $\Omega_{A} \neq \Omega_{D}$ only at the end of Sec. IV.

\section{The TASEP with a single inhomogeneity: A brief review}

Before presenting the details of our theoretical treatment of the TASEP/LK system in the presence of a bottleneck, and to gain some intuitive understanding of the underlying physics, it is convenient to outline the properties of the simple TASEP perturbed by a localized inhomogeneity $[7,10]$. Here we consider the TASEP with a defect at site $k$ where the hopping rate is $q<1$.

Consider the LD phase, where the system is "diluted" and the particles are well separated. In such a "low traffic" situation, one does not expect any macroscopic effects arising from the presence of the bottleneck. One rather expects a local peak in the density profile [see Fig. 3(a)]: $\rho_{i \neq k}=\alpha$ and $\rho_{k}=\alpha+h$ (LD phase), where $h$ is the height of the local jump imposed by the defect. Since an exclusion process without coupling to a bulk reservoir has a spatially constant current $j_{i}=\alpha(1-\alpha)$, one finds $h=h(\alpha, q)=\alpha(1-q) / q$. Thus, the height of the density peak increases with $\alpha$ and $q^{-1}$. This can certainly not happen without bound. In fact, $h$ cannot exceed $h_{\max }=1-2 \alpha$. This may be seen as follows. If $h>h_{\max }$, there would be a site $i_{1}$ such that $\rho_{i_{1}}<\alpha+h_{\max }=1-\alpha$ and $\rho_{i_{1}+1}$ $>\alpha+h_{\max }$. This case has to be discarded as it contradicts current conservation: $j_{i_{1}}=\rho_{i_{1}}\left(1-\rho_{i_{1}}\right)<j_{i}=\alpha(1-\alpha)$. Therefore, for given $\alpha$ and $\beta$, there is a critical value of $q_{\alpha}^{*}$ below which the local peak is no longer a possible solution. The critical $q_{\alpha}^{*}$ follows from the requirement that the height of the peak cannot exceed $h_{\max }$, i.e., $h\left(\alpha, q_{\alpha}^{*}\right)=h_{\max }$. One thus finds 


$$
q_{\alpha}^{*}=\frac{\alpha}{1-\alpha} .
$$

Equivalently, if one keeps $q$ fixed, a local peak is obtained for

$$
\alpha \leqslant \frac{q}{1+q}=\alpha_{c} .
$$

It directly follows from the underlying particle-hole symmetry that the same reasoning holds in the HD phase ( $\alpha$ $>\beta$ and $\beta<1 / 2$ ). There the system is so "packed" that the bottleneck is only responsible for a local dip in the density profile: $\rho_{i \neq k}=1-\beta$ and $\rho_{k}=1-\beta-h(\beta, q)$, with $h\left(\beta, q_{\beta}^{*}\right)$ $<h_{\max }^{\prime}=1-2 \beta$. Thus, in the HD phase, a local dip is only possible for

$$
q_{\beta}^{*} \equiv \frac{\beta}{1-\beta}<q
$$

or, keeping $q$ fixed, for

$$
\beta \leqslant \frac{q}{1+q}=\beta_{c} .
$$

We conclude that, for a fixed value of $q$, there are critical values for the entrance and exit rates above which the defect leads to a jump in the density profile. In contrast to the local density perturbation ("spike") for small $\alpha$ and $\beta$, this is a macroscopic effect and does not vanish in the thermodynamics limit (while the spike width scales as $1 / N$ ).

Denoting, respectively, by $\rho^{L}$ and $\rho^{R}$ the densities on the left and right of the inhomogeneity, one expects a sharp step profile displaying a jump at the defect position

$$
\rho^{L}=\frac{1}{2}+\delta \quad \text { and } \quad \rho^{R}=\frac{1}{2}-\delta .
$$

Thus, the current through the defect is

$$
j_{k}=q \rho_{k}\left(1-\rho_{k+1}\right)=q\left[\frac{1}{2}+\delta\right]\left[1-\left(\frac{1}{2}-\delta\right)\right],
$$

while the current flowing through the bulk of the track reads

$$
j_{i \neq k}=\left(\frac{1}{2}+\delta\right)\left(\frac{1}{2}-\delta\right) \text {. }
$$

As the current is conserved, these expressions should coincide, which gives the jump in the density profile

$$
2 \delta=\frac{1-q}{1+q} .
$$

This is an important quantity, which measures the strength of the defect: $\delta=0$ when $q=1$ and $\delta=1 / 2$ for $q=0$.

Thus, for the density profile, one finds

$$
\rho^{L}=\frac{1}{1+q} \quad \text { and } \quad \rho^{R}=\frac{q}{q+1}
$$

while the $q$-dependent current flowing through the entire system, playing the role of an effective (bottleneck-induced) $\mathrm{MC}$, reads
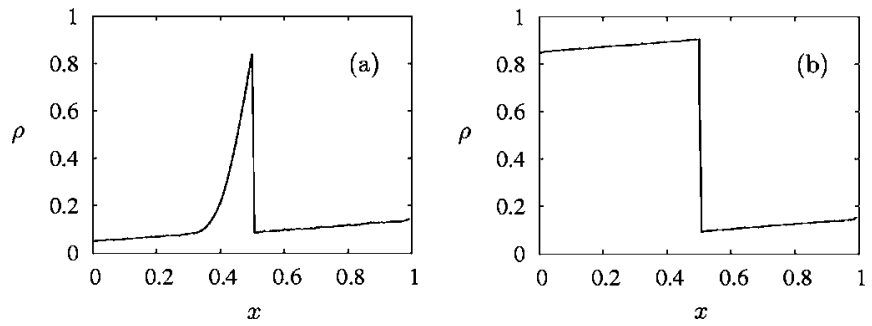

FIG. 4. Transition between spike (a) and step (b) for the TASEP/LK with defect. Stochastic simulations are performed on systems of size $N=128$ with parameters $q=0.1, x_{d}=0.5, \Omega=0.1$, (a) $(\alpha, \beta)=(0.05,0.8),(\mathrm{b})(\alpha, \beta)=(0.8,0.8)$.

$$
j_{d}^{*}=\frac{q}{(1+q)^{2}} \leqslant \frac{1}{4} \text {. }
$$

Since there is a jump and the density profile is flat on both sides of the defect, one may effectively split the systems into two parts connected by current conservation (see Fig. 5 and Refs. $[10,44,45])$. The effective exit rates of the left subsystem and the entrance of the right one are therefore

$$
\alpha_{\text {eff }}=\beta_{\text {eff }}=\frac{q}{1+q} .
$$

It follows from this discussion that for the TASEP, only the MC phase is affected by the presence of a bottleneck, while the LD and HD phases remain unaltered. The resulting phase diagram therefore still displays the same topological features (with three phases: LD, HD, and MC) as in the homogeneous case, with the lines $\alpha=\beta=1 / 2$, delimiting the MC phase, lowered to $\alpha=\alpha_{c}$ and $\beta=\beta_{c}$.

\section{EFFECTIVE MEAN-FIELD THEORY}

In this section, we describe an effective mean-field theory for the nonequilibrium steady state of the TASEP/LK in the presence of a bottleneck.

As for the TASEP perturbed by the presence of a localized defect, there are regions of the parameter space where the inhomogeneity affects the system only locally (Fig. 4). In other regions, the defect has macroscopic effects and is said to be relevant (see Fig. 3). In this case, the properties of the system are studied by splitting the lattice at the defect site $k$ into two subsystems (again termed $L$ and $R$ ) and performing a continuum limit (see Fig. 5). In such a limit, the position of the defect becomes $x_{d} \equiv \lim _{N \rightarrow \infty} k / N$, and the density can be written as

$$
\rho(x)=\left\{\begin{array}{ll}
\rho^{L}(x), & 0 \leqslant x \leqslant x_{d} \\
\rho^{R}(x), & x_{d}<x \leqslant 1
\end{array} .\right.
$$

In the presence of a bottleneck, the current is locally conserved for the same reasons as in the TASEP/LK system [34] [essentially because the attachment/detachment rates scale as in Eq. (5)] 


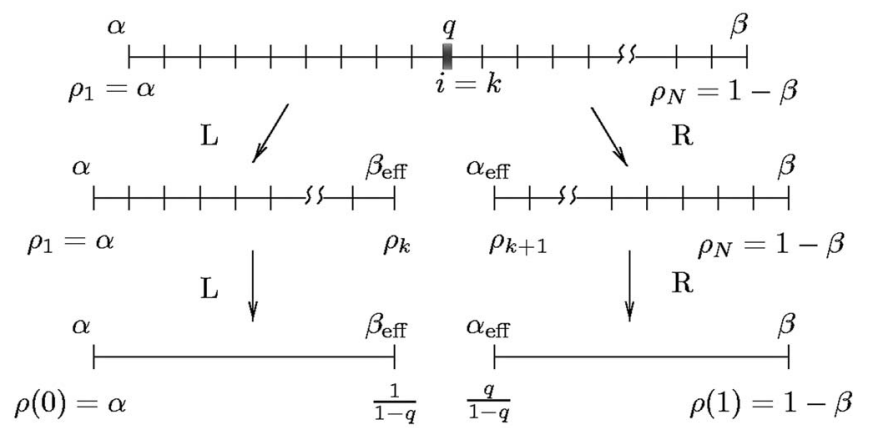

FIG. 5. Schematic representation of the division into two subsystems allowing to apply a mean-field theory. The last step (bottom of the figure), illustrates the continuum limit that we are considering (see the main text).

$$
j_{i+1}-j_{i}=\frac{1}{N}\left[\Omega_{A}-\left(\Omega_{A}+\Omega_{D}\right) \rho_{i+1}\right] \underset{N \rightarrow \infty}{\rightarrow} 0 .
$$

This allows coupling of both subsystems $R$ and $L$ along the same lines as for the simple TASEP perturbed by an isolated inhomogeneity, with effective right and left boundaries given by Eq. (25).

Suppose now that we are in the parameter range where the defect dominates the current and density profile. Then we may argue as in the previous section and obtain for the current at the defect site

$$
j_{k}=j_{k+1}=\frac{q}{(1+q)^{2}}
$$

and the corresponding densities

$$
\rho_{k}=\frac{1}{1+q}, \quad \rho_{k+1}=\frac{q}{1+q} .
$$

Again this implies a defect-induced jump in the density of magnitude

$$
2 \delta \equiv \rho_{k}-\rho_{k+1}=\frac{1-q}{1+q} .
$$

A key difference to our previous discussion of a pure TASEP in Sec. II is that these properties apply only locally in the vicinity of the defect. In the continuum limit, the density profile is linear [see Eq. (10)] and has to match the boundary conditions given by Eqs. (29). Hence the density profile $\rho_{d}(x)$ imposed by the defect reads

$$
\rho_{d}(x)=\left\{\begin{array}{ll}
\Omega\left(x-x_{d}\right)+\frac{1}{1+q}, & (L) \\
\Omega\left(x-x_{d}\right)+\frac{q}{1+q}, & (R)
\end{array} .\right.
$$

Since the mean-field current-density relationship is given by Eq. (11), this immediately implies for the current

$$
j_{d}=\left(\Omega\left|x-x_{d}\right|+\frac{q}{1+q}\right)\left(\frac{1}{1+q}-\Omega\left|x_{d}-x\right|\right) .
$$

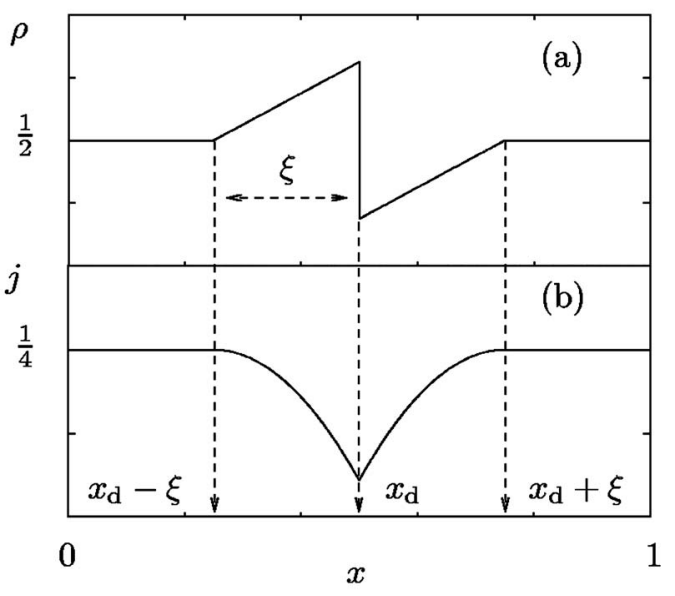

FIG. 6. Sketch of the signature of the strong defect (zigzag shape) in the density (a) and current (b) profile.

In stark contrast to the simple TASEP, here the current is a space-dependent quantity. As illustrated in Fig. 6, the signature of a defect is the depletion on a macroscopic scale of the current profile [Fig. 6(b)]. Correspondingly, the density displays a "zigzag" shape with a jump at $x_{d}$ and a linear profile in its vicinity as expressed in Eq. (31) [see Fig. 6(a)].

At $x=x_{d} \pm \xi$, the current imposed by the defect reaches the maximal value $j^{*}=1 / 4$. Thus, the depletion or screening length $\xi$ induced by the bottleneck is the solution of $\rho_{d}\left(x_{d} \pm \xi\right)=\rho^{*}=1 / 2$ and reads

$$
\xi=\frac{\delta}{\Omega} .
$$

The defect is thus screened best for a strong coupling to the reservoir $(\Omega \gg \delta)$ and, of course, if the inhomogeneity is weak, i.e., $\delta \rightarrow 0$.

Since the screening length increases with the strength of the defect it can even become larger than the length of the subsystems. This happens when $\xi$ is larger than the distance to either one of the boundaries. According to the conditions $x_{d}-\xi<0$ and $x_{d}+\xi>1$, this happens when $\delta>\max \left(\delta_{1}, \delta_{2}\right)$ (and fixed $\Omega$ ) or, equivalently, when $\Omega<\min \left(\Omega_{1}, \Omega_{2}\right.$ ) (with fixed $q$ ), where

$$
\begin{gathered}
\delta_{1}=\Omega x_{d} \text { and } \delta_{2}=\Omega\left(1-x_{d}\right), \\
\Omega_{1}=\frac{\delta}{x_{d}} \text { and } \Omega_{2}=\frac{\delta}{1-x_{d}} .
\end{gathered}
$$

If $\delta<\min \left(\delta_{1}, \delta_{2}\right)$ the screening length is shorter than both lengths of the subsystems. When the location of the defect is not centered, two additional cases arise: for $\delta_{1}<\delta<\delta_{2}\left(\delta_{2}\right.$ $<\delta<\delta_{1}$ ) the screening length stays within the subsystem $R$ $(L)$, while it is larger than the size of the sublattice $L(R)$.

In the TASEP and in the TASEP/LK the maximal current that can flow through the system is a constant $j^{*}=1 / 4$. For the TASEP with a defect, that value is lowered to $j_{d}^{*}=q /(1$ $+q)^{2}$ [see Eq. (24)]. As the current is space dependent and locally conserved, in the TASEP/LK with an inhomogeneity, the maximal flow of particles that can be transported through 

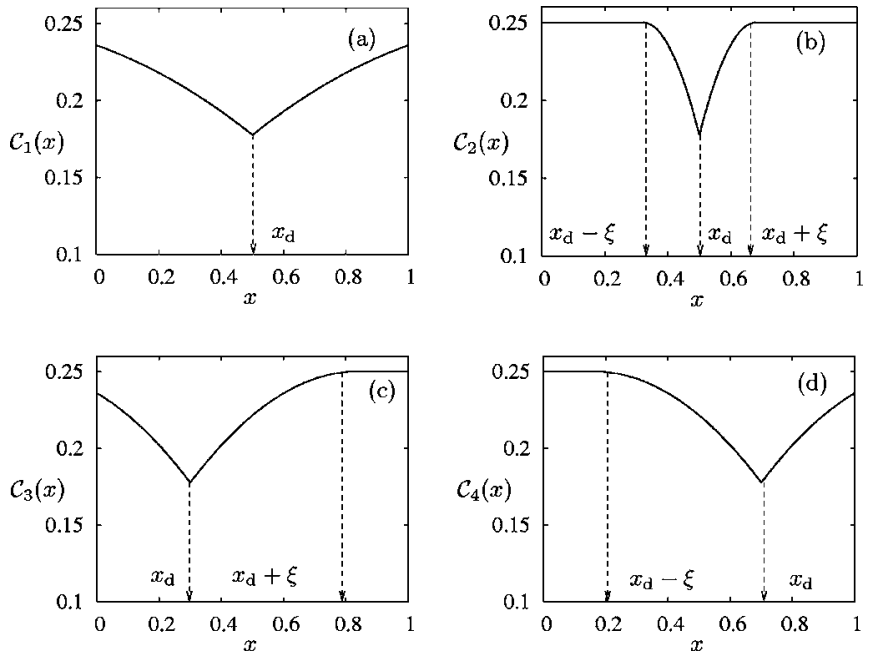

FIG. 7. The four typical carrying capacity profiles $\mathcal{C}(x)$ displayed by the system (parameters $\Omega=\{0.3,1.5,0.5,0.5\}$, $\left.x_{d}=\{0.5,0.5,0.7,0.3\}, q=0.3\right)$. Depending on the defect strength $q$, on the position $x_{d}$ of the defect, and on the rate $\Omega$, the defectimposed current $j_{d}$ combines in four different ways with the maximal current $j^{*}$. Each of these profiles induces a topologically distinct phase diagram (see the text).

the bulk of the system varies spatially and is limited by the condition of having to match the value $j_{d}$ [Eq. (32)] in the vicinity of the defect. This suggests naming such a quantity the carrying capacity $\mathcal{C}(x)$ of the system.

The more drastic effect of the bottleneck appears when its strength satisfies $\delta>\max \left(\delta_{1}, \delta_{2}\right)$. In this case, the defect screens the entire system and, as shown in Fig. 7(a), the carrying capacity reads

$$
\mathcal{C}(x)=\mathcal{C}_{1}(x)=j_{d}(x) .
$$

In intermediate cases, when the screening length covers part of the system, one has three possible scenarios illustrated in Figs. 7(b)-7(d), namely,

$$
\begin{gathered}
\mathcal{C}(x)=\mathcal{C}_{2}(x)=\left\{\begin{array}{cc}
j_{d}(x), & \left|x-x_{d}\right|<\xi \\
j^{*}, & \text { else }
\end{array},\right. \\
\mathcal{C}(x)=\mathcal{C}_{3}(x)=\left\{\begin{array}{cc}
j_{d}(x), & 0<x<x_{d}+\xi \\
j^{*}, & x_{d}+\xi \leqslant x<1
\end{array},\right. \\
\mathcal{C}(x)=\mathcal{C}_{4}(x)=\left\{\begin{array}{cc}
j^{*}, & 0<x<x_{d}-\xi \\
j_{d}(x), & x_{d}-\xi \leqslant x<1
\end{array} .\right.
\end{gathered}
$$

For each possible carrying capacity of Fig. 7, one obtains different phase diagrams. All these scenarios are discussed in the next section.

To determine the global current and density profiles from the carrying capacity, one compares the latter with the currents imposed by the open boundaries

$$
\begin{gathered}
j_{\alpha}=(\alpha+\Omega x)(1-\alpha-\Omega x), \\
j_{\beta}=[\beta+\Omega(1-x)][1-\beta-\Omega(1-x)],
\end{gathered}
$$

corresponding to the left and right density profiles

$$
\begin{gathered}
\rho_{\alpha}(x)=\Omega x+\alpha, \\
\rho_{\beta}(x)=\Omega(x-1)+(1-\beta) .
\end{gathered}
$$

As $\mathcal{C}(x)$ acts as an effective maximal current in the bulk, $j_{\alpha}(x)$ and $j_{\beta}(x)$ cannot exceed its value. Actually, as for the TASEP (where $\mathcal{C}=1 / 4$ ), the entrance and exit boundaries only matter on the macroscopic part of the system where they impose currents smaller than $\mathcal{C}(x)$. Thus, by matching $j_{\alpha}(x)$ and $j_{\beta}(x)$ with the carrying capacity, one determines boundaries separating boundary-induced phases and mixed ones.

\section{RESULTS: PHASE DIAGRAM OF THE TASEP/LK IN THE PRESENCE OF A BOTTLENECK}

In this section we discuss the four possible scenarios arising for each carrying capacity presented above. We explicitly construct the density profiles and the phase diagrams when the screening length is larger than the size of the two subsystems [carrying capacity $\mathcal{C}_{1}(x)$ ] and when $\xi$ is shorter than the size of the two subsystems [carrying capacity $\mathcal{C}_{2}(x)$, as well as the cases characterized by $\mathcal{C}_{3}(x)$ and $\left.\mathcal{C}_{4}(x)\right]$. In the last subsection we extend the results obtained so far for the case of equal attachment and detachment rates $\left(\Omega_{A}=\Omega_{D}\right)$ to the more general situation $\Omega_{A} \neq \Omega_{D}$.

\section{A. Large screening length: The case $\mathcal{C}_{1}(x)$}

Let us first consider the case where the carrying capacity is entirely determined by the defect, i.e., $\mathcal{C}_{1}(x)=j_{d}(x)$. In this situation, sketched in Fig. 7(a), the bottleneck is strong enough and always imposes a current $j_{d}(x)<j^{*}$. Then we may distinguish between three cases depending on the magnitude of the current imposed by the left and right boundary (see Fig. 8).

There are two extreme cases: the entrance and exit boundary currents $j_{\alpha}$ and $j_{\beta}$ exceed the carrying capacity $\mathcal{C}_{1}(x)$; the current through the system settled at the value $j_{d}(x)$ imposed by the defect (this is the situation for the left subsystem presented as case 3 in Fig. 8). Thus, the density exhibits the piecewise profile given by Eq. (31). As the current is independent of the left and right boundaries, this phase is termed pure bottleneck phase (BP). In contrast, for low entrance and exit rates one recovers the TASEP/LK density profile perturbed by a local spike or a dip (see the previous section). This is presented as case 1 in Fig. 8.

In intermediate regions of the parameter space, a situation like the one presented as case 2 in Fig. 8 appears. Similarly to what happens for the homogeneous TASEP/LK, when the densities $\rho_{\alpha}, \rho_{\beta}$, and $\rho_{d}$ cannot be matched continuously, shocks form in the density profile and then we have coexistence of several phases. This can happen either on the left or right subsystem. The positions of the shocks follow from the local conservation of the current, i.e., $j_{\alpha}\left(x_{w}^{L}\right)=j_{d}\left(x_{w}^{L}\right)$ (on $L$ ) and $j_{\beta}\left(x_{w}^{R}\right)=j_{d}\left(x_{w}^{R}\right)$ (on $R$ ). With Eqs. (32)-(41), one finds

$$
x_{w}^{L}=\frac{1}{2}\left(x_{d}+\frac{1}{\Omega}\left\{\frac{1}{2}-\delta-\alpha\right\}\right),
$$



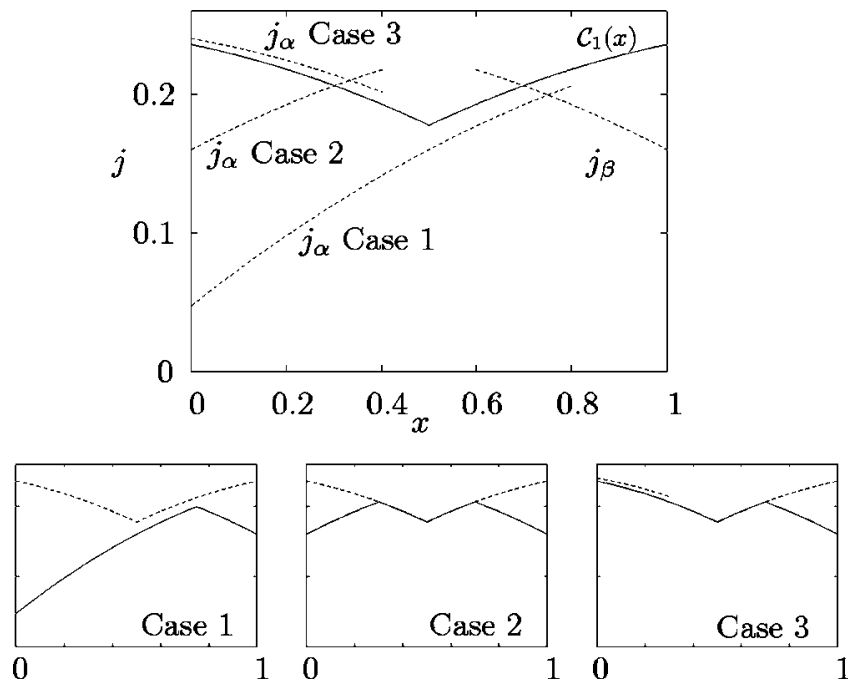

FIG. 8. Construction of the current profile. Top: For given carrying capacity $\mathcal{C}_{1}(x)$ (solid line) and fixed exit rate $\beta=0.2$, various scenarios arise when the entrance rate $\alpha=\{0.05,0.2,0.6\}$ is varied. Bottom: Three different profiles are shown in the small graphs (solid line: global current profile, dashed line: defect and boundary currents) emerging from three different left boundary conditions. Parameters are $x_{d}=1 / 2, q=0.3, \Omega=0.3, \beta=0.2, \alpha=\{0.05,0.2,0.6\}$.

$$
x_{w}^{R}=\frac{1}{2}\left(1+x_{d}+\frac{1}{\Omega}\left\{\beta+\delta-\frac{1}{2}\right\}\right) .
$$

The conditions for having domain walls within the two subsystems are $0<x_{w}^{L}<x_{d}$ and $x_{d}<x_{w}^{R}<1$. This translates into the following conditions for the entrance and exit rates:

$$
\begin{gathered}
\frac{1}{2}-\delta-\Omega x_{d}<\alpha<\frac{1}{2}-\delta+\Omega x_{d}, \\
\frac{1}{2}-\delta-\Omega\left(1-x_{d}\right)<\beta<\frac{1}{2}-\delta+\Omega\left(1-x_{d}\right) .
\end{gathered}
$$

These conditions allow the identification of two values of the boundary rates $\alpha_{c}^{-}$and $\beta_{c}^{-}$for which the domain wall enters, respectively, the left or right subsystem, and two values $\alpha_{c}^{+}$and $\beta_{c}^{+}$for which the domain wall exits the left or right subsystem;

$$
\begin{gathered}
\alpha_{c}^{ \pm} \equiv \frac{1}{2}-\delta \pm \Omega x_{d}, \\
\beta_{c}^{ \pm} \equiv \frac{1}{2}-\delta \pm \Omega\left(1-x_{d}\right) .
\end{gathered}
$$

Within the range defined by these critical rates, a shock is localized in each subsystem. At values above $\alpha_{c}^{-}$and $\beta_{c}^{-}$the defect becomes relevant and at least part of the density and current profile is dictated by the defect. This suggests naming the corresponding regions of the parameters space bottleneck phases.

Let us now construct the density profiles in the various bottleneck phases focusing first on the left subsystem. Depending on the entrance rate, one distinguishes two cases: (i) The density profile corresponding to case 2 in Fig. 8, arising when $\alpha_{c}^{-}<\alpha<\alpha_{c}^{+}$, is presented in Fig. 9(a) (numerical simu-
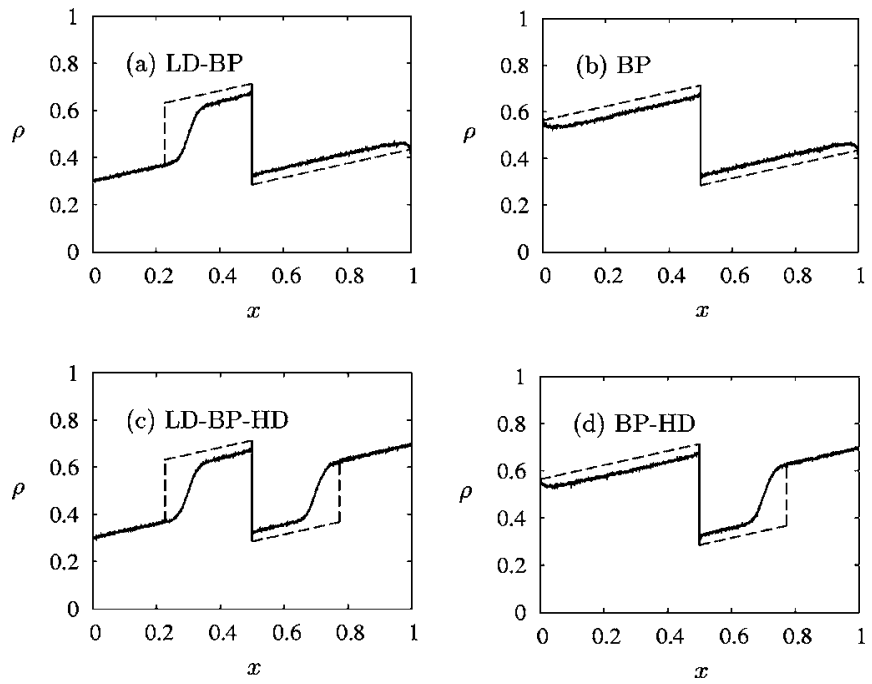

FIG. 9. Examples of density profiles in the bottleneck phases for a carrying capacity $\mathcal{C}(x)=\mathcal{C}_{1}(x)$. Stochastic simulations (continuous line) are compared to analytical mean-field predictions (dashed line). The system size is $N=4096$ and the parameters are $q=0.3$, $x_{d}=0.5, \Omega_{D}=0.3$, LD-BP: $(\alpha, \beta)=(0.3,0.6)$, BP: $(\alpha, \beta)=(0.6,0.6)$, LD-BP-HD: $(\alpha, \beta)=(0.3,0.3)$ and BP-HD: $(\alpha, \beta)=(0.6,0.3)$.

lations are discussed later). Here, $j_{\alpha}$ intersects the left branch of $\mathcal{C}_{1}(x)$ at $x_{w}^{L}$, where a domain wall forms. Then the density (of the left subsystem) reads

$$
\rho^{L}(x)= \begin{cases}\rho_{\alpha}(x), & 0<x<x_{w}^{L} \\ \rho_{d}(x), & x_{w}^{L}<x<x_{d} .\end{cases}
$$

This coexistence phase is called LD-BP since it is characterized by the coexistence of a low density and a bottleneck phase. (ii) The density profile corresponding to case 3 in Fig. 8, arising for $\alpha>\alpha_{c}^{+}$, is shown in Fig. 9(b). Here, $j_{\alpha}$ is always above the left branch of $\mathcal{C}_{1}(x)$ and the defect imposes an effective high-density phase (called simply BP) corresponding to the maximal current $j_{d}$ on subsystem $L$. The corresponding density profile reads $\rho^{L}(x)=\rho_{d}(x)$.

One proceeds in a similar way for the right subsystem $(R)$. When $\beta_{c}^{-}<\beta<\beta_{c}^{+}$, there is coexistence between highdensity (boundary-induced) and an effective low-density (defect-induced) phases, called BP-HD [see Fig. 9(d)]. This results in a domain wall at $x_{w}^{R}$ and in the density

$$
\rho^{R}(x)= \begin{cases}\rho_{d}(x), & x_{d}<x<x_{w}^{R} \\ \rho_{\beta}(x), & x_{w}^{R}<x<1 .\end{cases}
$$

When $\beta>\beta_{c}^{+}$, the defect dominates and imposes an effective low-density phase called BP, where the density is $\rho^{R}(x)$ $=\rho_{d}(x)$.

Considering the whole system, by combining the above mixed phases (on $R$ and $L$ ) we obtain the following four bottleneck phases: 


\begin{tabular}{c|cc} 
Left $\downarrow /$ Right $\rightarrow$ & BP-HD & BP \\
\hline LD-BP & LD-BP-HD & LD-BP \\
BP & BP-HD & BP
\end{tabular}

The emergence of these four defect-dominated mixed phases is the most dramatic effect of the bottleneck when $\delta>\max \left\{\delta_{1}, \delta_{2}\right\}$. We have checked the predictions of our MF theory against stochastic numerical simulations (following the Bortz-Kalos-Lebowitz scheme for kinetic Monte Carlo [46]) and, as shown in Fig. 9, have found good agreement (both qualitative and quantitative) with the predictions of the MF theory. Of course, due to finite-size effects, boundary layers form in the vicinity of the shocks and "soften" the transition in the density profile. When the system size is increased, the accuracy of the MF theory improves and the density profile displays a sharp jump. In addition to boundary layers [as already noted in Ref. [7]] for the simple TASEP perturbed by one defect, in the bottleneck phases, one notes a slight but systematic deviation from the MF predictions [see, e.g., Figs. 9(b) and 9(d)]. This effect is due to correlations not taken into account by the mean-field approximation and is long range, scaling as the inverse of the distance to the defect [7].

The various phases are summarized in the $\alpha / \beta$ phase diagram of Fig. 10. In this figure, because $x_{d}=1 / 2$ and $\Omega_{A}$ $=\Omega_{D}=\Omega$, the particle-hole symmetry (which still holds in the presence of the bottleneck) results in the invariance of the phase diagram with respect to the line $\alpha=\beta$.

As can be seen from the phase diagram of Fig. 10, the bottleneck-induced mixed phases (LD-BP-HD, LD-BP, BP$\mathrm{HD}$, and $\mathrm{BP}$ ) occupy the upper right part of the diagram (the shadowed region in Fig. 10). Only at the borders of the phase diagram, corresponding to particularly low (high) entrance (exit) rates, one recovers the same phases as in the defect-

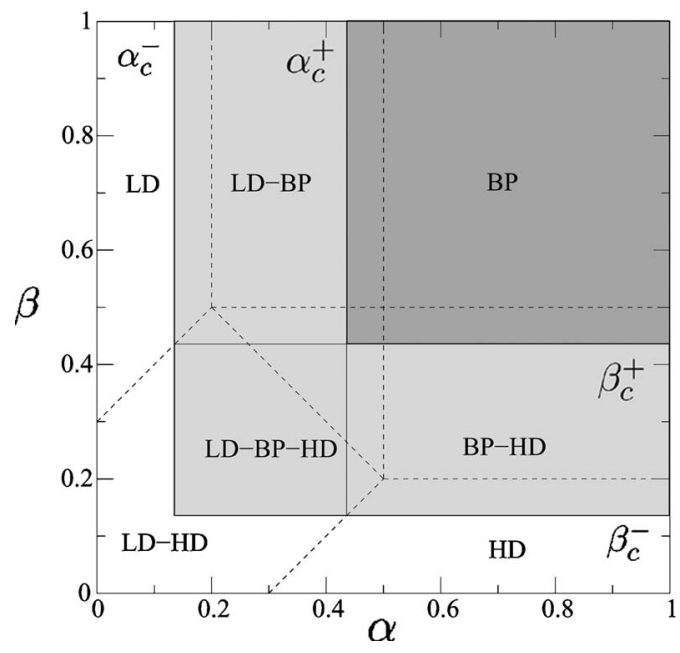

FIG. 10. Phase diagram for $\Omega=0.3, q=0.4$, and $x_{d}=1 / 2$, i.e., for the carrying capacity $\mathcal{C}_{1}(x)$ (large screening length). Continuous lines are the phase boundaries introduced by the defect; dashed lines are the phase boundaries already present in the model without bottleneck. The shadowed region indicates the bottleneck phases where the defect is relevant, the darkest one highlights the pure bottleneck phase (for the meaning of the different phases see text). free TASEP/LK model (there the defect is irrelevant). For $\alpha>\alpha_{c}^{+}$and $\beta>\beta_{c}^{+}$, i.e., in the right top corner of the phase diagram (the darkest shadowed region in Fig. 10), the entire system is in a pure bottleneck phase. By tuning the strength of the defect $\delta$ and the on-off parameter $\Omega$, the phase boundaries can be shifted to recover the short screening length case (discussed in the following) and eventually the usual TASEP/LK behavior (when the defect is irrelevant). On the other hand, by increasing the strength of the defect (or by reducing the on-off rate) the phase boundaries move toward the axes of the phase diagram and can even merge with them. Actually, this occurs for a defect strength $\widetilde{\delta}$. Above this threshold, the whole $\alpha / \beta$ phase diagram is characterized by bottleneck phases (the homogeneous LD, HD, and LD-HD phases are squeezed out of the diagram). The critical strength $\widetilde{\delta}$ is therefore the maximum between the value determined by the conditions $\alpha_{c}^{-}=0$ and $\beta_{c}^{-}=0$, which leads to

$$
\widetilde{\delta}=\max \left\{\frac{1}{2}-\delta_{1}, \frac{1}{2}-\delta_{2}\right\} .
$$

Note that for a too strong defect, $\delta>\widetilde{\delta}$, the control of the system from the boundaries is lost.

\section{B. Short screening length}

We now consider the case where the screening length is short so that the carrying capacity reaches the value $j^{*}$ $=1 / 4$ in the bulk of the system.

\section{Symmetric screening}

We first consider the case illustrated in Fig. 7(b) where the carrying capacity loses the trace of the defect in the system at a distance larger than the screening length

$$
\mathcal{C}_{2}(x)=\left\{\begin{array}{ll}
j_{d}(x), & \left|x-x_{d}\right|<\xi \\
\frac{1}{4}, & \text { else }
\end{array} .\right.
$$

This situation arises when $\delta<\min \left\{\delta_{1}, \delta_{2}\right\}$ (with fixed $\Omega$ ) or, equivalently, when $\Omega>\max \left\{\Omega_{1}, \Omega_{2}\right\}$ (with fixed $\delta$ ). This carrying capacity corresponds to the richest case in terms of new bottleneck phases, due to the profile of $\mathcal{C}_{2}$ characterized by four distinct regions.

As in the previous situation, the bottleneck is relevant and induces new mixed phases when $\alpha>\alpha_{c}^{-}$and $\beta>\beta_{c}^{-}$, where these critical values are again given by Eqs. (48). Elsewhere in the parameter space, the homogeneous TASEP/LK profiles locally perturbed by a spike (or a dip) are recovered.

When the system is driven above its carrying capacity $(\alpha, \beta>1 / 2)$, it exhibits the current profile given in Eq. (53), corresponding to a density profile

$$
\rho^{L}(x)=\left\{\begin{array}{ll}
\rho^{*}, & x_{d}-x>\xi \\
\rho_{d}(x), & x_{d}-x<\xi
\end{array} .\right.
$$

As the current profile of Eq. (53) reaches the MC value, contrary to the case of a large screening length, this is no longer a pure bottleneck phase but corresponds to a MCBP-MC phase (see the dark shadowed region in Fig. 12). 
Between these two extremal situations, more intricate mixed phases appear. As in the previous case, phases characterized by shocks appear when the boundary currents match $j_{d}$.

Contrary to the previous situation, where $j_{d}$ covered the whole subsystem, now the defect-imposed current extends up to a distance equal to the screening length from the bottleneck. Hence, shocks can emerge only in a macroscopic region, $x_{d}-\xi<x_{w}^{L}<x_{d}$ and $x_{d}<x_{w}^{R}<x_{d}+\xi$, in the vicinity of the defect. These conditions translate into new critical values of the entrance and exit rates, namely,

$$
\alpha_{c}^{\prime}=\frac{1}{1+q}-\Omega x_{d}, \quad \beta_{c}^{\prime}=\frac{1}{1+q}-\Omega\left(1-x_{d}\right) .
$$

As a new scenario, here the boundary currents can reach the upper-bound $j^{*}=1 / 4$ on the subsystems $L$ and $R$ when $j_{\alpha}\left(x^{L}\right)=j_{\beta}\left(x^{R}\right)=1 / 4$. With Eqs. (40) and (41), one finds that this occurs at

$$
x^{L}=\frac{1-2 \alpha}{2 \Omega}, \quad x^{R}=1-\frac{1-2 \beta}{2 \Omega} .
$$

We note that these quantities are independent of the properties of the defect, which is thus screened. Let us consider a density profile that exemplifies the richness of this case. When $\alpha_{c}^{\prime}<\alpha<\alpha^{*}=1 / 2$ the resulting current $j_{\alpha}(x)$ saturates at the value $1 / 4$, while the density reads $\rho^{L}(x)=1 / 2$ for $x^{L}$ $<x<x_{d}-\xi$. Similarly, on the subsystem $R$, when $\beta_{c}^{\prime}<\beta$ $<\beta^{*}=1 / 2$ and the current $j_{\beta}(x)$ saturates at the value $1 / 4$, the density is $\rho^{L}(x)=1 / 2$ for $x_{d}+\xi<x<x^{R}$. Within the range of the screening length, $x_{d}-\xi<x<x_{d}+\xi$, the carrying capacity and the current flowing through the system coincide with $j_{d}(x)$ and the density is given by Eq. (31). Summarizing, in this case the density profile is piecewise and one distinguishes five regions

$$
\rho(x)= \begin{cases}\rho_{\alpha}(x), & 0<x<x^{L} \\ \rho^{*}, & x^{L}<x<x_{d}-\xi \\ \rho_{d}(x), & x_{d}-\xi<x<x_{d}+\xi \\ \rho^{*}, & x_{d}+\xi<x<x^{R} \\ \rho_{\beta}(x), & x^{R}<x<1\end{cases}
$$

This case, denoted LD-MC-BP-MC-HD, is illustrated in Fig. 11 and corresponds to the coexistence of a low-density and high-density (on $L$ and $R$, respectively), two maximal currents (one in both subsystems), and a bottleneck-induced mixed phase.
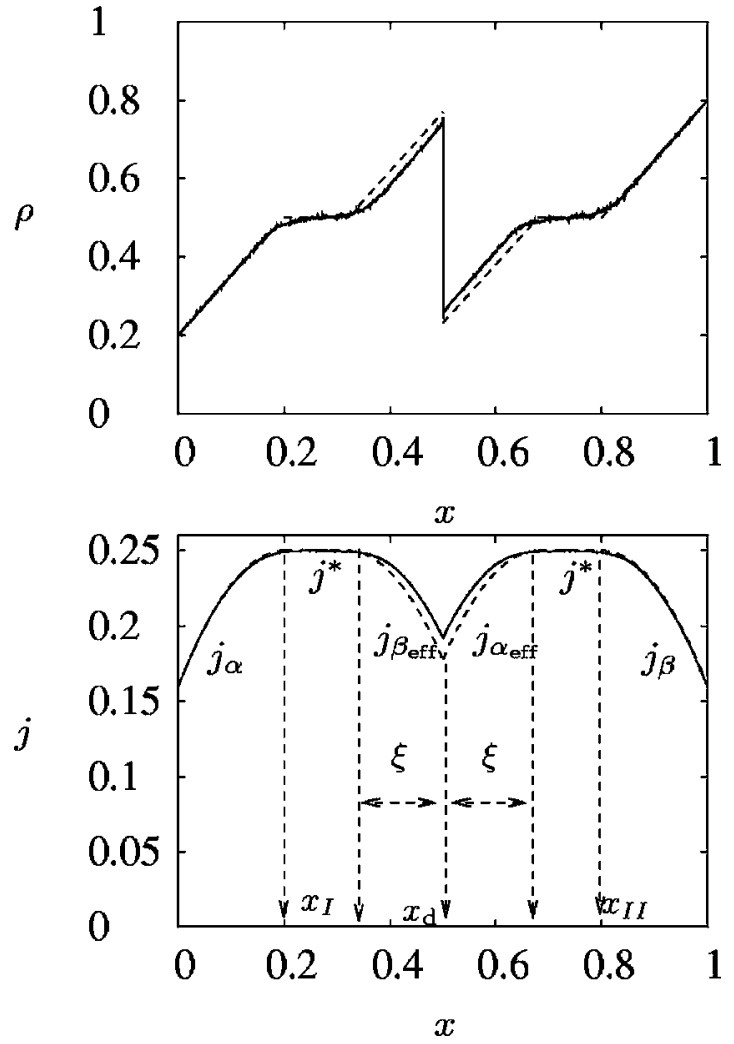

FIG. 11. Examples of density profile (top) and current (bottom) for the system in the LD-MC-BP-MC-HD phase: the defect is relevant and the carrying capacity is $\mathcal{C}_{2}(x)$. Results of stochastic simulations (continuous line) are compared to analytical mean-field predictions (dashed line). The system size is $N=4096, K=1, \Omega=\Omega_{D}$ $=1.5, q=0.3,(\alpha, \beta)=(0.2,0.2)$, and $x_{d}=1 / 2$ : one can clearly distinguish the various phases and note a discontinuity in the density profile in the proximity of the defect, while the current profile is continuous.

The three possible scenarios for the bottleneck phases on the two sublattices results in nine mixed phases on the whole system, as summarized in Table I.

We have also checked our MF predictions against stochastic numerical simulations, as illustrated in Fig. 11. Again, we have found qualitative and quantitative agreement. The small deviations from the MF theory observed in Fig. 11 can be explained along the above discussion on the role of the correlations and finite-size effects.

The $\alpha / \beta$ phase diagram corresponding to a system with a carrying capacity $\mathcal{C}_{2}(x)$ is shown in Fig. 12 and characterized by the transition lines corresponding to the critical values $\alpha_{c}^{-}, \alpha_{c}^{\prime}, \alpha^{*}$ and $\beta_{c}^{-}, \beta_{c}^{\prime}, \beta^{*}$. The nine bottleneck-induced

TABLE I. Bottleneck phases for the case $\mathcal{C}(x)=\mathcal{C}_{2}(x)$. For each phase, the label (1-9) refers to a given region of the (shadowed part) of the phase diagram represented in Fig. 12.

\begin{tabular}{c|ccc}
\hline \hline Left $\downarrow /$ Right $\rightarrow$ & BP-HD & BP-MC-HD & BP-MC \\
\hline LD-BP & LD-BP-HD $^{7}$ & LD-BP-MC-HD & LD-BP-MC \\
LD-MC-BP & LD-MC-BP-HD $^{8}$ & LD-MC-BP-MC-HD & LD-MC-BP-MC \\
MC-BP & MC-BP-HD & MC-BP-MC-HD & MC-BP-MC \\
\hline \hline
\end{tabular}




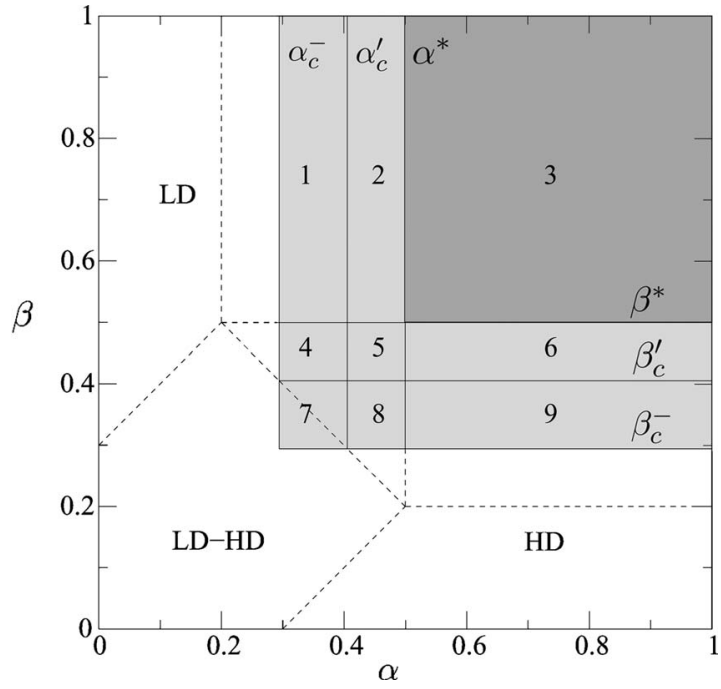

FIG. 12. Phase diagram for $\Omega=0.3, x_{d}=1 / 2$, and $q=0.8$, i.e., for the carrying capacity $\mathcal{C}_{2}(x)$ (short screening length). Continuous lines are the phase boundaries introduced by the defect; dashed lines are the phase boundaries already present in the model without bottleneck. The shadowed region indicates the bottleneck phases where the defect is relevant; the darkest one highlights the bottleneck phase independent of the entrance $(\alpha)$ and exit $(\beta)$ rates (see the text and Table I).

phases appear in the core of the phase diagram (shadowed region in Fig. 12). Contrary to the phase diagram obtained with $\mathcal{C}=\mathcal{C}_{1}$, instead of a pure bottleneck phase, here one finds a MC-BP-MC coexistence phase, which is independent of the entrance and exit boundaries (right top corner of Fig. 12). This phase, obtained when $\alpha>\alpha^{*}$ and $\beta>\beta^{*}$, is the "bottleneck analogous" of the MC phase in the homogeneous TASEP/LK system.

\section{Asymmetric (partial) screening}

To conclude the study of the case $\Omega_{A}=\Omega_{D}=\Omega$, let us briefly consider the scenarios where the system displays a carrying capacity of type $\mathcal{C}_{3}(x)$ or $\mathcal{C}_{4}(x)$. This is possible when the defect is not at the center of the system. In fact, the carrying capacity of the system is $\mathcal{C}_{3}(x)$ when $\delta_{1}<\delta<\delta_{2}$ and is $\mathcal{C}_{4}(x)$ when $\delta_{2}<\delta<\delta_{1}$. As one can infer from the profile of $\mathcal{C}(x)=\mathcal{C}_{3}(x)$ [see Fig. 7(c)], on the subsystem $L(R)$ this case is identical to that discussed for $\mathcal{C}(x)=\mathcal{C}_{1}(x)[\mathcal{C}(x)$ $\left.=\mathcal{C}_{2}(x)\right]$. Therefore, it directly follows from the above table that there are six new phases for the system displaying a carrying capacity $\mathcal{C}_{3}(x)$. The latter are summarized in the following table:

\begin{tabular}{c|ccc} 
Left $\downarrow /$ Right $\rightarrow$ & BP-HD & BP-MC-HD & BP-MC \\
\hline LD-BP & $\begin{array}{c}\text { LD-BP-HD } \\
\text { LD-BP-MC-HD }\end{array}$ & LD-BP-MC \\
BP & BP-HD & BP-MC-HD & BP-MC
\end{tabular}

Similarly, six bottleneck phases are also obtained for a carrying capacity $\mathcal{C}(x)=\mathcal{C}_{4}(x)$, as it is clear from the table below.

\begin{tabular}{c|cc} 
Left $\downarrow /$ Right $\rightarrow$ & BP-HD & BP \\
\hline LD-BP & LD-BP-HD & LD-BP \\
LD-MC-BP & LD-MC-BP-HD & LD-MC-BP \\
MC-BP & MC-BP-HD & MC-BP
\end{tabular}

The corresponding phase diagrams directly follow from those discussed above for the carrying capacities $\mathcal{C}_{1}(x)$ and $\mathcal{C}_{2}(x)$.

\section{Topological features}

The above discussion has shown that the sole presence of a localized bottleneck is responsible for the emergence of new (sub)phases and drastic topological changes of the $\alpha / \beta$ phase diagram. Here we aim to discuss further the important structural changes induced by the bottleneck in the density and current profiles of the TASEP/LK by considering the $\alpha / \delta$ phase diagram (see Fig. 13).

We have already seen that the most appealing properties of the phase diagrams of the model under consideration are the new bottleneck (sub)phases. As summarized in Figs. 10 and 12, a large portion of the $\alpha / \beta$ phase diagram is dominated by the defect properties. The phase boundaries separating the usual phases from the bottleneck (i.e., $\alpha=\alpha_{c}^{-}$and $\beta=\beta_{c}^{-}$) are straight lines since they depend only on the detachment rate and on the strength and position of the defect, but not on the entrance and exit rates.

In the simple TASEP, the transitions between the usual phases and the bottleneck phases can be considered as discontinuous, since the influence of the defect changes abruptly from a local peak to a global step (see Fig. 3). In the case of TASEP/LK with bottleneck, a shock continuously enters the system and the transition can be considered indeed

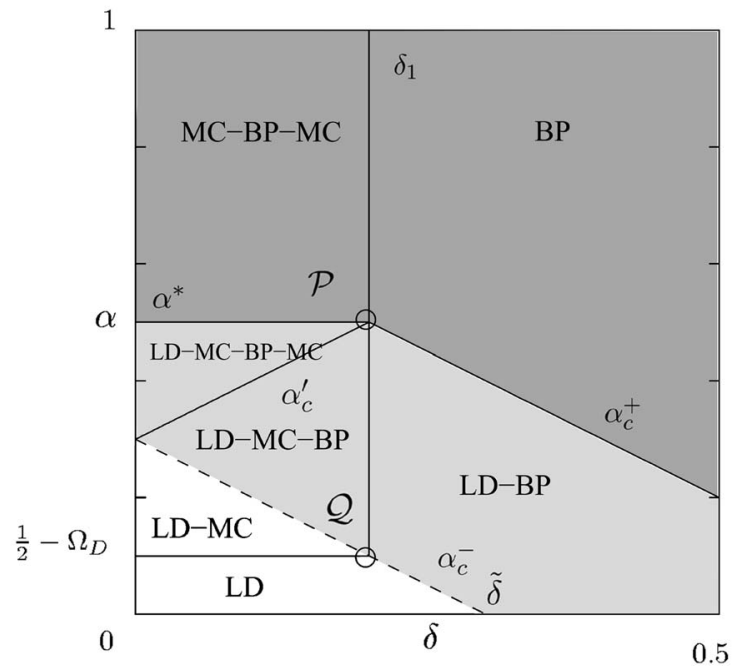

FIG. 13. Cut of the phase diagram in $\delta$ and $\alpha$ (parameters: $\Omega$ $\left.=0.4, x_{d}=1 / 2, \beta>1 / 2\right)$. Solid lines identify usual phase transitions, while the dashed line identifies the peculiar transition between relevant and irrelevant defect. The multiple coexistence points $\mathcal{P}$ and $\mathcal{Q}$ are shown in the graph (see the main text). 
continuous, as it was in the simple TASEP/LK (the dashed line in Fig. 13).

The phase boundaries $\alpha_{c}^{+}\left(\beta_{c}^{+}\right)$, for $\delta>\delta_{1}\left(\delta>q_{2}\right)$, and $\alpha^{*}=1 / 2\left(\beta^{*}=1 / 2\right)$, for $\delta<\delta_{1}\left(\delta<\delta_{2}\right)$, separate the point where the density profile is dominated by the defect from the one where the boundary currents still play a role. Considering the position of the domain walls $x_{w}^{L}$ and $x_{w}^{R}$ as the order parameters, the transitions are all continuous. A similar transition is also identified by the boundary $\alpha_{c}^{\prime}\left(\beta_{c}^{\prime}\right)$ since the matching point (no longer a shock) $x^{L}\left(x^{R}\right)$ enters the system continuously.

The physical modifications in the system following the transition between a carrying capacity $\mathcal{C}_{1}(x)$ and $\mathcal{C}_{2}(x)$ can be observed if we plot the cut of the phase diagram in $\delta$ and $\alpha$ (choosing $\beta>1 / 2$ ), as done in Fig. 13. In this graph, the line $\alpha_{c}^{-}$identifies the transition between relevant and irrelevant defect, while $\alpha_{c}^{+}$, $\alpha_{c}^{\prime}$, and $\alpha^{*}=1 / 2$ are the transitions between bottleneck phases. The line $\alpha=1 / 2-\Omega_{D}$ was present in the pure TASEP/LK. The line $\delta=\delta_{1}$ identifies the continuous transition between the two different carrying capacities, $\mathcal{C}_{1}(x)$ and $\mathcal{C}_{2}(x)$. There are two relevant points in the phase diagram where different phases coexist, indicated with $\mathcal{P}$ (five phases) and $\mathcal{Q}$ (four phases) in Fig. 13.

\section{Results and phase diagrams when $\Omega_{A} \neq \Omega_{D}$}

In this section we discuss the case where the attachment and detachment rates differ, $\Omega_{A} \neq \Omega_{D}$. This is mathematically more tedious, as for the homogeneous TASEP/LK model at MF level, one needs to solve Eqs. (8) and (12), the solution of which implies multivalued (Lambert) functions with two real branches [34]. Introducing the binding constant $K$ $\equiv \Omega_{A} / \Omega_{D} \neq 1$, the Langmuir isotherm $\rho_{I}$ reads $\rho_{I} \equiv K /(K$ $+1)$. Here, having split the problem in two subsystems one has to consider the equation

$$
\left(2 \rho^{L, R}-1\right) \partial_{x} \rho^{L, R}(x)=(K+1) \Omega_{D}\left[\rho_{I}-\rho^{L, R}(x)\right],
$$

again supplemented with the (subsystems) boundary conditions Eqs. (12) and (25)

$$
\begin{gathered}
\rho^{L}(0)=\alpha, \quad \rho^{L}\left(x_{d}\right)=\frac{1}{1+q}, \\
\rho^{R}\left(x_{d}\right)=\frac{q}{q+1}, \quad \rho^{R}(1)=1-\beta .
\end{gathered}
$$

We refer the readers to Ref. [34] for a detailed mathematical treatment of this kind of equation, and report our results for the phase diagram and density profiles of the TASEP/LK model in the presence of a bottleneck when $\Omega_{D} \neq \Omega_{A}$ (i.e., $K \neq 1$ ). As for the homogeneous model, one can take advantage of the underlying particle-hole symmetry to restrict the discussion to the case $K>1$ [34]. Except for the mathematical treatment of the MF bulk equation, we follow the same lines as in the case $K=1$. Again, one has to distinguish the case where the carrying capacity coincides with the current imposed by the defect $\left[\mathcal{C}(x)=j_{d}(x)\right]$ from the situation where $\mathcal{C}(x)$ reaches the maximal current value $j_{K}^{*}$. While for $K=1$ the maximal current available in the bulk is $j^{*}=1 / 4$, here $j_{K}^{*}(x) \leqslant j^{*}$ is a nonconstant space-dependent quantity. When

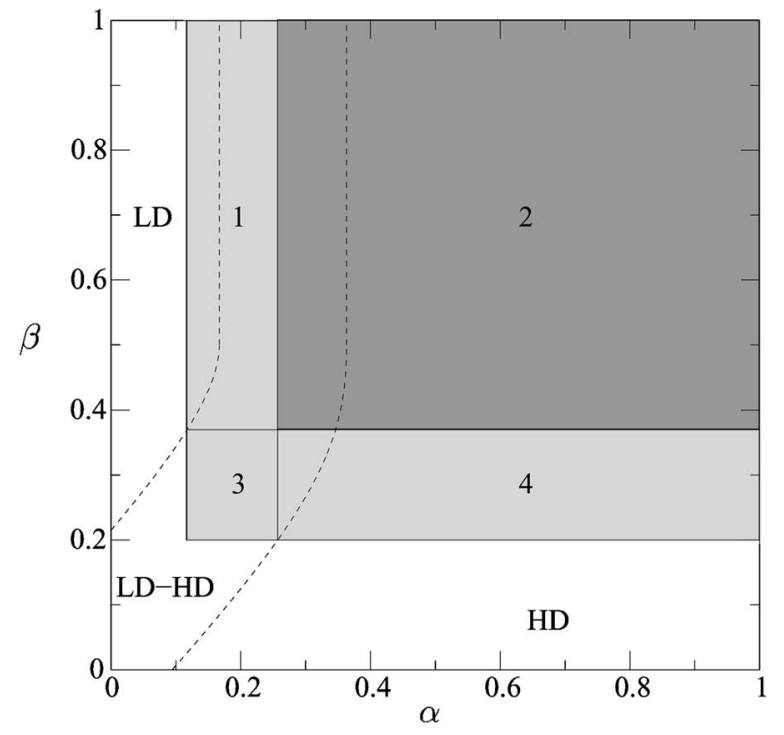

FIG. 14. Phase diagram for $K=2, \Omega_{D}=0.1, x_{d}=1 / 2$, and $q$ $=0.3$, i.e., for the $\mathcal{C}_{1}(x)$-like carrying capacity. Continuous lines are the phase boundaries introduced by the defect (BP); dashed lines are the phase boundaries already present in the model without bottleneck. The shadowed region indicates the bottleneck phases where the defect is relevant; the darkest one highlights the pure bottleneck phase. The numbers stem for the different phases: LD-BP (1), BP (2), LD-BP-HD (3), and BP-HD (4). For the description of the phases see the text and Figs. 15.

$\mathcal{C}(x)=j_{d}(x)$, except some topological asymmetries, one essentially recovers the same phase diagram as in the $K=1$ situation when the carrying capacity is of $\mathcal{C}_{1}(x)$ type: as illustrated in Fig. 14, the new bottleneck (sub)phases are BP, MC-BP, LD-P, and LD-BP-HD [see Fig. 15 (left) for an example of current and density profiles]. Again, we notice that the core of the phase diagram is entirely determined by the defect (the shadowed region in Fig. 14), which is also responsible for a pure BP for sufficiently high rates $\alpha$ and $\beta$.

On the other hand, when $K \neq 1$ it follows from the analytical solution of the above-mentioned MF equation that there is only one (finite) screening length on the subsystem $R$. In fact, it turns out that the left branch of $j_{d}(x)$ can never reach the maximal current $j_{K}^{*}(x)$ on the subsystem $L$ (so, for $K \neq 1$, there is no finite screening length on the subsystem $L$ ): the screening length may only be shorter than the length of the subsystem $R$, with the point $x_{d}+\xi<1$. It follows that carrying capacities of types $\mathcal{C}_{2}(x)$ and $\mathcal{C}_{4}(x)$ are topologically prohibited (even in the asymmetric case $x_{d} \neq 1 / 2$ ) when $K \neq 1$.

In contrast to the case $K=1$, as in general the current $j_{\beta}(x)$ cannot reach the value $j_{K}^{*}(x)$, the carrying capacity of type $\mathcal{C}_{3}(x)$ gives rise to four bottleneck phases (instead of six as for the case $K=1$ ), namely, the LD-BP-HD, LD-BP-MC, BP$\mathrm{HD}$, and BP-MC. An example of BP-MC current and density profiles is shown in Fig. 15 (right).

\section{CONCLUSION}

This work has been devoted to the study of the influence of a bottleneck (pointwise disorder) on the stationary prop- 

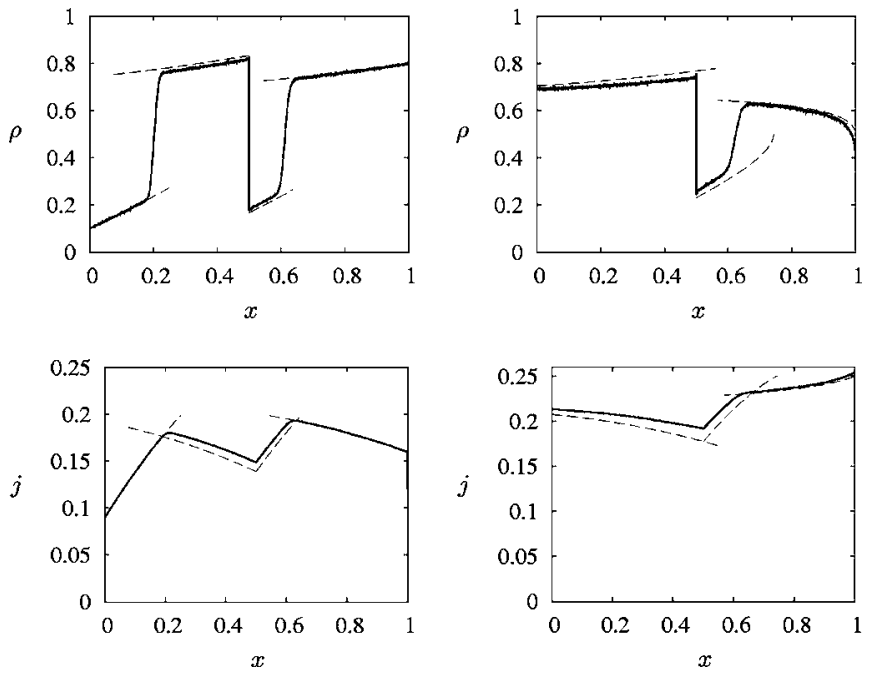

FIG. 15. Two examples of density profile and current in the LD-BP-HD for the $\mathcal{C}_{1}(x)$-like carrying density capacity (left) and BP-MC phase for the $\mathcal{C}_{3}(x)$ (right). Stochastic simulations (continuous line) are compared to analytical mean-field predictions (dashed line). The system size is $N=4096$ and the parameters are $q=0.2$, $x_{d}=1 / 2, K=2, \Omega_{D}=0.3,(\alpha, \beta)=(0.1,0.2) \quad(\mathrm{left}) ; q=0.3, x_{d}=1 / 2$, $K=2, \Omega_{D}=0.3,(\alpha, \beta)=(0.8,0.8)$ (right).

erties of a biologically inspired stochastic transport model obtained by coupling two paradigmatic equilibrium and nonequilibrium processes. Namely, we have considered the competition between the TASEP and Langmuir kinetics (LK) in the presence of open boundaries and a bottleneck, which locally slows down any incoming particles. The current and density profiles in the nonequilibrium steady state have been investigated analytically via an effective mean-field theory built on splitting the lattice into two subsystems. Our analytical results were checked against numerical (Monte Carlo) simulations.

As a consequence of the competition between the TASEP and LK dynamics, the effects of a single bottleneck in the TASEP/LK model are much more dramatic than in the simple TASEP [10], or the so-called $\ell$-TASEP (TASEP for extended objects) [14], where a localized defect was shown to merely shift some transition line in the phase diagram, but do not affect its topology. Here, new and mixed phases induced by the bottleneck have been obtained.

As a key concept of our analysis, we have introduced the carrying capacity, which is defined as the maximal current that can flow through the bulk of the system. In contrast to the simple TASEP, the spatial dependence of the current, caused by the Langmuir kinetics, makes the carrying capacity nontrivial: The defect depletes the current profile within a distance that we called screening length. This quantity increases with the strength of the defect and decreases with the attachment-detachment rates. The competition between the current imposed at the boundaries and the one limited by the defect determines the density profiles and the ensuing phase diagram. When the boundary currents are dominant, the phase behavior of the defect-free system is recovered. Also, above some critical entrance and exit rates, the system transports the maximal current, independently of the boundaries. Between these two extreme situations, we have found several coexistence phases, where the density profile exhibits stable shocks and kinks. Indeed, above some specific parameter values the phase diagram is characterized by bottleneck phases. Depending on the screening length imposed by the defect, which can cover the entire system or part of it, different phase diagrams arise. The latter are characterized by four, six, or nine bottleneck phases, which have been quantitatively studied within our mean-field theory.

The analysis carried out in this work can be straightforwardly extended to many variants of the TASEP/LK model. As an example, let us mention the case of a lattice gas where dimers (modeling the usual two heads of molecular motors) would move as bound entities according to the $\ell$-TASEP (with $\ell=2$ ) [47] and could experience Langmuir-type on-off kinetics. Recently, such a system has been studied (without disorder) within an appropriate mean-field-like scheme [36]. The pointwise version of this system could be investigated along the same lines described in the present work.

In addition to direct extensions, we think that the method outlined in this paper could pave the way to study the TASEP/LK models in more "realistic" and biophysically relevant situations, as in the presence of clusters of competing defects or quenched sitewise randomness.

\section{ACKNOWLEDGMENTS}

The authors are grateful to T. Reichenbach, A. Parmeggiani, and T. Franosch for useful discussions. M.M. gratefully acknowledges the support of the German Alexander-vonHumboldt Foundation (Grant No. IV-SCZ/1119205 STP).
[1] J. Ziman, Models of Disorder: The Theoretical Physics of Homogeneously Disordered Systems (Cambridge University Press, Cambridge, 1979).

[2] R. Stinchcombe, Dilute Magnetism, Phase Transition and Critical Phenomena (Academic, New York, 1983), Vol. 7.

[3] R. Stinchcombe, J. Phys.: Condens. Matter 14, 1473 (2002).

[4] Z. Cshaók and T. Vicsek, J. Phys. A 27, L591 (1994).

[5] J. Krug and P. Ferrari, J. Phys. A 29, L465 (1994).

[6] J. Krug, Braz. J. Phys. 30, 97 (2000).
[7] S. A. Janowsky and J. L. Lebowitz, Phys. Rev. A 45, 618 (1992).

[8] G. Tripathy and M. Barma, Phys. Rev. Lett. 78, 3039 (1997).

[9] M. Evans, J. Phys. A 30, 5669 (1997).

[10] A. B. Kolomeisky, J. Phys. A 31, 1152 (1998).

[11] K. M. Kolwankar and A. Punnoose, Phys. Rev. E 61, 2453 (2000).

[12] M. Ha, J. Timonen, and M. den Nijs, Phys. Rev. E 68, 056122 (2003). 
[13] N. Mirin and A. B. Kolomeisky, J. Stat. Phys. 110, 811 (2003).

[14] L. B. Shaw, A. B. Kolomeisky, and K. H. Lee, J. Phys. A 37, 2105 (2004).

[15] L. B. Shaw, J. P. Sethna, and K. H. Lee, Phys. Rev. E 70, 021901 (2004).

[16] T. Chou and G. Lakatos, Phys. Rev. Lett. 93, 198101 (2004).

[17] R. J. Harris and R. B. Stinchcombe, Phys. Rev. E 70, 016108 (2004).

[18] C. Enaud and B. Derrida, Europhys. Lett. 66, 83 (2004).

[19] M. R. Evans, T. Hanney, and Y. Kafri, Phys. Rev. E 70, 066124 (2004).

[20] R. Juhász, L. Santen, and F. Iglói, Phys. Rev. Lett. 94, 010601 (2005).

[21] B. Derrida, Phys. Rep. 301, 65 (1998).

[22] G. Schütz, Exactly Solvable Models in Many-body Systems, Phase Transition and Critical Phenomena (Academic Press, London, 2001), Vol. 19.

[23] J. Howard, Mechanics of Motor Proteins and the Cytoskeleton (Sinauer Associates, Inc., Sunderland, 2001)

[24] A. Ylzid and P. Selvin, Trends Cell Biol. 15, 112 (2005).

[25] G. Cappello (private communication).

[26] C. MacDonald, J. Gibbs, and A. Pipkin, Biopolymers 6, 1 (1968).

[27] C. MacDonald and J. Gibbs, Biopolymers 7, 707 (1969).

[28] R. Lipowsky, S. Klumpp, and T. M. Nieuwenhuizen, Phys. Rev. Lett. 87, 108101 (2001).

[29] A. Parmeggiani, T. Franosch, and E. Frey, Phys. Rev. Lett. 90, 086601 (2003).

[30] A. Ebneth, R. Godemann, K. Stamer, S. Illenberger, B. Trinczek, E.-M. Mandelkow, and E. Mandelkow, J. Cell Biol. 143, 777 (1998)
[31] L. Goldstein, Proc. Natl. Acad. Sci. U.S.A. 98, 6999 (2001).

[32] We consider that the bulk is the whole system except the first and last sites. Thus, in the bulk the sites are labeled $i=2, \ldots$, $N-1$.

[33] For $q>1$ one expects only variations of the dynamics but not of the stationary properties; the phase diagram is the same as for the defect-free model.

[34] A. Parmeggiani, T. Franosch, and E. Frey, Phys. Rev. E 70, 046101 (2004).

[35] H. Hinsch, R. Kouyos, and E. Frey, Traffic and Granular Flow 2005, edited by P. Pierobon, T. Pöschel, R. Kühne, M. Schreckenberg, and D. E. Wolf (Springer, Berlin, 2006).

[36] P. Pierobon, T. Franosch, and E. Frey, Phys. Rev. E (to be published), e-print cond-mat/0603385.

[37] B. Derrida, E. Domany, and D. Mukamel, J. Stat. Phys. 69, 667 (1992).

[38] B. Derrida, M. Evans, V. Hakim, and V. Pasquier, J. Phys. A 26, 1493 (1993).

[39] B. Derrida and M. Evans, J. Electron. Imaging 3, 311 (1993).

[40] B. Derrida and M. Evans, J. Phys. A 32, 4833 (1999).

[41] G. Schütz and E. Domany, J. Stat. Phys. 72, 277 (1993).

[42] J. Krug, Phys. Rev. Lett. 67, 1882 (1991).

[43] A. B. Kolomeisky, G. Schütz, E. Kolomeisky, and J. Straley, J. Phys. A 31, 6911 (1998).

[44] J. Brankov, N. Pesheva, and N. Bunzarova, Phys. Rev. E 69, 066128 (2004).

[45] E. Pronina and A. B. Kolomeisky, J. Stat. Mech.: Theory Exp. 7, 159 (2005).

[46] A. Bortz, M. Kalos, and J. Lebowitz, J. Comput. Phys. 17, 10 (1975).

[47] L. B. Shaw, R. K. P. Zia, and K. H. Lee, Phys. Rev. E 68, 021910 (2003) 